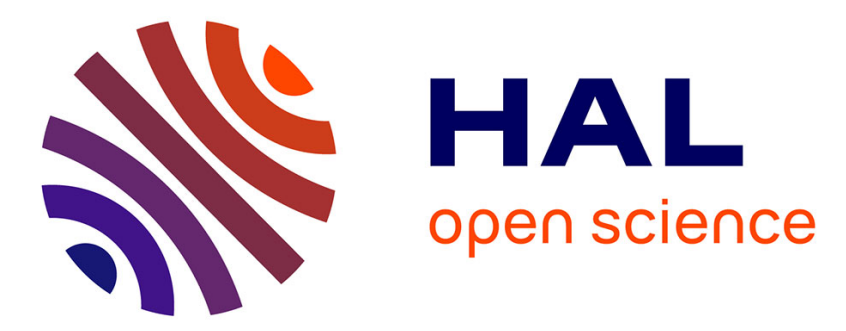

\title{
An alternative to Lyapunov Exponent as Damage Sensitive Feature
}

Antoine Clément, S. Laurens

\section{To cite this version:}

Antoine Clément, S. Laurens. An alternative to Lyapunov Exponent as Damage Sensitive Feature. Smart Materials and Structures, 2011, 20 (2), pp.025017. 10.1088/0964-1726/20/2/02501 . hal00557282

\section{HAL Id: hal-00557282 \\ https://hal.science/hal-00557282}

Submitted on 18 Jan 2011

HAL is a multi-disciplinary open access archive for the deposit and dissemination of scientific research documents, whether they are published or not. The documents may come from teaching and research institutions in France or abroad, or from public or private research centers.
L'archive ouverte pluridisciplinaire HAL, est destinée au dépôt et à la diffusion de documents scientifiques de niveau recherche, publiés ou non, émanant des établissements d'enseignement et de recherche français ou étrangers, des laboratoires publics ou privés. 


\title{
An alternative to Lyapunov Exponent as Damage Sensitive Feature
}

\author{
Antoine Clément
}

Stéphane Laurens

Université de Toulouse; UPS, INSA; LMDC (Laboratoire Matériaux et Durabilité des Constructions); 135, avenue de Rangueil; F-31 077 Toulouse Cedex 04, France

E-mail: Stephane.laurens@insa-toulouse.fr

\begin{abstract}
.
When using Lyapunov exponents as damage sensitive feature (DSF) in vibration based structural health monitoring, the computation time represents an important issue for real time applications. To overcome this problem, a new feature, the Jacobian Feature Vector (JFV), is proposed based on the same algorithm but allowing faster computation. Whereas for Lyapunov exponents calculation, the trajectories in the state space are followed for more than a thousand time steps, the new feature is formed by the Jacobian matrix of the dynamics after few time steps. The new feature and the Lyapunov exponents are tested on two case studies : a mass/spring/damper model and a laboratory three story structure. Both the DSF are showing a good performance in detecting damage for the numerical study, even if the Lyapunov exponents present a slightly better ability. Regarding the experimental data, Lyapunov exponents failed to detect damage while the JFV is clearly able to reveal structural changes. Therefore, the JFV offers a robust alternative to Lyapunov exponents allowing to save computation time.
\end{abstract}

PACS numbers:

Submitted to: Smart Mater. Struct. 


\section{Introduction}

Structural Health Monitoring (SHM) consists in studying information which is generated by the structure to be investigated. The paradigm of non linear dynamical systems offers some interesting abilities for this purpose. From a general point of view, the scientific objective is focused on the detection of novelty in monitoring data. Among the various features available to describe dynamical systems, the Lyapunov Exponents (LE) are often proposed to detect changes in the system behavior.

A dynamical system can be represented by the evolution of its trajectory in its state space. Based on this representation, it is possible to calculate several invariants which characterize the dynamical system. Among them, Lyapunov exponents express the rate of divergence (or convergence) of trajectories initially close. If the system changes, due for example to an evolution of its parameters, the invariant property guaranties that the Lyapunov Exponents will deviate as well.

In the field of SHM, it is necessary to propose features that are sensitive to structural changes so that damage can be detected. The discrimination of damaged state is done by comparison with a reference baseline, generated under undamaged condition. The use of feature based on state space representation for time series analysis is limited in SHM. However, several studies explore the potential of this approach.

A first possibility is to excite the structure with a chaotic signal and to analyse the response in the state space. This method is used in several studies $[1,2]$ working on laboratory test structures, with different original features based on attractor geometry changes. Nichols et al [3] show that it is possible to apply the same paradigm on a structure using ambient vibrations. A comparison of several metrics calculated on trajectory evolution for chaotic and broadband excitations has been carried out by Olson et al [4].

The sensibility of Lyapunov exponents to damage has also been studied. They are used in the work reported by Ghafari et al [5] in which the chaotic vibrations of rolling element bearings are investigated. Bearing failure is detected by the calculation of the largest Lyapunov Exponent.

Some studies have also proven the ability of Lyapunov Exponent to detect damage even in a non chaotic system. Livingston et al [6] simulate cracks in a bridge FEM model excited by a traffic load model. The largest Lyapunov exponent is calculated along the bridge deck and is able to detect and locate the cracks. An application in a real structure have been carried out by Casciati et Casciati [7] on a monumental arch (masonry specimen) before and after the repair of several major cracks. Two excitations are considered : hammer impact, and ambient vibrations. The method combines all the sensors to form the state space. It shows a good ability in detecting damage and even in locating cracks, as long as major non-stationarities are removed.

The last cited studies focus on the largest Lyapunov exponent or only the positives

ones, but none of them uses the full spectrum. The main reasons are linked to the difficulty to estimate the negative Lyapunov exponents and to the high computation 
time. Though, when the aim is to find a Damage Sensitive Feature (DSF), even if the negative exponents estimations do not reflect exactly the real physical ones, they still may contain some information concerning the evolution of the system that can be useful in a comparative purpose.

This paper proposes a novel DSF based on Lyapunov exponent calculation algorithm : the Jacobian Feature Vector (JFV). It has to overcome the limitation of calculation time and has to minimize the loss of information about the dynamical system. After an introduction to the theoretical background leading to the definition of the JFV and LE, their practical calculation is presented. A comparison of the two DSF is then conducted on two case studies : 4 dof mass/spring/damper numerical model, and a laboratory structure. At last, a discussion on the performance of the DSF is proposed.

\section{Theoretical background and DSF definition}

The definition of the new DSF is closely related to the meaning of the LE.

Lyapunov exponents are linked to the predictability of the dynamical system studied. Viewed in the state space, let $\delta(0)<<1$ be the distance between two nearby trajectories. After $m$ time steps $(m>>1)$, the distance will be

$$
\delta(m) \approx \delta(0) e^{\lambda m}
$$

with $\lambda$ the maximum Lyapunov exponent [8]. If the time is infinite, the ergodic theorem of Oseledec [9] proves the existence and uniqueness of $\lambda$, whatever the initial point chosen. The Eq.1 illustrates the fact that if a small perturbation is imposed to the system, the rate of amplification of this perturbation is the Lyapunov exponent. If $\lambda$ is positive, the trajectories will diverge making the system unpredictable after some time. This kind of behavior characterizes chaotic systems, which are sensitive to small perturbation.

The calculation of the integer spectrum is done by following two initially close trajectories by the linearized dynamics [10].

Let $X(k) \in \mathbb{R}^{n}$ be a point moving in a $n$-dimensional space at discrete time $k=1,2, \ldots, N$. The evolution of $X(k)$ defines the trajectory and satisfies

$$
X(k+1)=F(X(k))
$$

with $F$ the dynamical system operator. If a nearby trajectory $Y(k) \in \mathbb{R}^{n}$ is taken and noting $\delta X_{i}=X(k+i)-Y(k+i)$, the first-order Taylor expansion of $F$ around $X(k)$ leads to

$$
\begin{aligned}
\delta X_{1} & =X(k+1)-Y(k+1) \\
& =F(X(k))-F(Y(k)) \\
& =J(X(k))[X(k)-Y(k)]+o\|X(k)-Y(k)\| \\
& =J(X(k)) \delta X_{0}+o\left\|\delta X_{0}\right\|
\end{aligned}
$$

with $J$, the Jacobian matrix of $F$. When the trajectories are followed for $L$ steps ahead, the product of $L$ Jacobian matrices needs to be calculated and it is noted $J^{L}\left(X_{k}\right)$ 
(Eq. 4).

$$
\begin{aligned}
\delta X_{L} & =J\left(X_{k}\right) \cdot J\left(X_{k+1}\right) \cdot \ldots \cdot J\left(X_{k+L-1}\right) \cdot \delta X_{0} \\
& =\left[\prod_{j=0}^{L-1} J\left(X_{k+j}\right)\right] \cdot \delta X_{0} \\
& =J^{L}\left(X_{k}\right) \cdot \delta X_{0}
\end{aligned}
$$

The Oseledec matrix, $J^{L}\left(X_{k}\right)$. $\left[J^{L}\left(X_{k}\right)\right]^{T}$, has eigenvalues noted $\Lambda_{i}, i=1 \ldots n$. Then the Lyapunov exponents are

$$
\lambda_{i}=\lim _{L \rightarrow \infty} \frac{1}{2 L} \ln \left(\Lambda_{i}\right)
$$

This equation (Eq. 5) implies that each trajectory needs to be followed for a very long time leading to :

- a very long computing time

- the hiding of every short irregularities of the trajectories.

These irregularities are the markers of damage induced, for example, by shocks in a loosened bolt, or by opening and closing of cracks. One is interested in detecting this local changes for SHM purpose. To highlight these irregularities, local Lyapunov exponents can be computed by following trajectories but only for a short time [11]. This approach has been more used to study the predictability of chaotic system than for SHM applications. But these irregularities should be visible directly on the Jacobian matrices since, in both cases (global or local), Lyapunov exponents represent the eigenvalues of the product of Jacobian matrices (Eq.5).

To preserve the potential sensitivity of local Lyapunov exponents and to provide a more simple approach, Jacobian matrix for short time evolution can be directly used to detect local distortions in trajectories. The components of the matrix are forming a new feature vector which will be referred to as the Jacobian Feature Vector (JFV).

\section{Practical implementation}

In experimental structural analysis, only a few degree of freedom of the system can be measured corresponding to each sensor location. Each measure is presented in the form of a time series. This section describes the algorithm applied on a single time series to obtain the Lyapunov spectrum and the JFV. The first step is to transform a scalar time series into a multi-dimensional time series to reconstruct the state space.

\section{State Space Reconstruction}

Based on the measure of an unique scalar time series $(x)=\{x(1), x(2), \ldots, x(N)\}$, Takens proves that it is possible to qualitatively reconstruct the attractor in a ndimensional state space by using delayed coordinates method [12]. This supposes that one single degree of freedom encloses enough information about the others. 
Theoretically, the delay, $\tau$, could be any entire number. In practice, it is common to choose $\tau$ long enough, to limit the redundancy between coordinates, and not too much, to keep some correlation. Once this choice is made, the state space vectors are formed, for $k=1, \ldots, n-\tau(n-1)$, as follows:

$$
X(k)=\{x(k), x(k+\tau), \ldots, x(k+(n-1) \tau)\}
$$

Two methods for evaluating the optimal time delay $\tau$ are commonly used, the first minimum of the mutual information function and the first zero of the autocorrelation function [13] . Then, one needs to find the proper embedding dimension, $n$, in which the attractor is completely unfolded. Brown et al suggest the method of false neighbors [14] which counts the number of neighbors which do not remains neighbors as the dimension increases. The stabilization of this number indicates that the embedding dimension is large enough.

Once $n$ and $\tau$ are selected, the time series is transformed into a collection of state space vectors according to Eq.6.

\section{JFV and Lyapunov exponents Calculation}

To evaluate the Lyapunov spectrum, the method described by Brown et al [14] is used. The first step consists in calculating the Jacobian matrix along a trajectory. For this purpose, a fiducial point $X(k)$ is chosen, and its $r$ nearest neighbors, noted $X_{\text {neigh }}^{r}(k)$, are selected. Difference vectors are formed according to Eq. 8.

$$
\begin{aligned}
& X_{\text {neigh }}^{r}(k)=\{X(i), i \in[1 ; N] \mid\|X(k)-X(i)\| \leq \epsilon \in \mathbb{R}\} \\
& \delta X_{\text {neigh }}^{r}(k)=\left\{X(k)-X(i) \mid X(i) \in X_{\text {neigh }}^{r}(k)\right\}
\end{aligned}
$$

To estimate the Taylor expansion of $F$, the best polynomial mapping, noted $T(X)$, between each of the $r$ perturbation vectors at time $k$ and its evolutions at time $k+T_{2}$ (Eq.9) is calculated by a least-square method. Then, the Jacobian matrix associated to the linear part of $T(X)$ is extracted.

$$
\begin{aligned}
\delta X_{\text {neigh }}^{i}\left(k+T_{2}\right)= & T\left(\delta X_{\text {neigh }}^{i}(k)\right) \\
& \text { with } i=0, \ldots, r
\end{aligned}
$$

The original approach uses a linear mapping, but the polynomial one is more suitable to estimate the negative exponents accurately [15].

The Jacobian matrix is estimated for $L T_{2}$ time steps, giving $J\left(X_{k+i T 2}\right), i=1, \ldots, L$.

Since the JVF has to reveal a local information, it is formed by the last Jacobian matrix for very few time steps. The $L$ values from 1 to 5 time steps are tested (Eq.10).

$$
J F V=J\left(X_{k+i T_{2}}\right)(:) \quad i \leq 5
$$

On the other hand, to find the Lyapunov spectrum, the eigenvalues of the product of $J\left(X_{k+i T 2}\right)$ for $i=1, \ldots, L$ need to be calculated (Eq.4). $L$ has to be at least 2000 to 
ensure the convergence of the limit (Eq.5). Since this product is very ill-conditioned, a $Q R$ decomposition method is recommended [16].

$$
\begin{aligned}
J\left(X_{k+(i+1) T_{2}}\right) \cdot Q(i)= & Q(i+1) \cdot R(i+1) \\
& \text { with } \quad i=0, \ldots, L-1
\end{aligned}
$$

Since $Q(i)$ is an orthogonal matrix and $R(i)$ an upper triangular one, the product of Eq. 4 is decomposed as

$$
J^{L}\left(X_{k}\right)=Q(L) \prod_{j=1}^{L} R(j)
$$

Finally, the Lyapunov exponents are expressed as

$$
\lambda_{i}=\frac{1}{d t L T_{2}} \sum_{j=L_{0}}^{L} \ln \left(R_{i i}(j)\right) \quad i=1, \ldots, n
$$

with $d t$ the sampling period (in seconds) of the original scalar time series. The sum should start at $L_{0} \neq 0$ to omit the first transient values.

For both Lyapunov exponents and JFV, the algorithm is repeated for several fiducial points across the attractor and the average of each DSF is taken.

\section{SHM and Novelty Detection}

For the long term monitoring of structures, damage can only be detected by comparison with a reference normal state acquired from various conditions [17]. For each condition, time series are collected thanks to vibration measurements and compressed into feature vectors such JFV and LE. To compare a new feature vector to the baseline vectors collected at the reference state, the Mahalanobis distance is an appropriate approach [18]. It gives the distance between a point and a set of points, taking into account the way the axis are populated. In statistics, it is commonly used to detect abnormal points in a data set. The advantage is that it provides a scalar feature from multivariate data. The Mahalanobis distance of a new feature vector $D S F_{i}$ (which can be either the Lyapunov exponents or the JVF) to a reference baseline characterized by a mean vector $\overline{D S F}$ and a covariance matrix $C_{D S F}$ is

$$
D_{i}^{2}=\left(D S F_{i}-\overline{D S F}\right)^{T}\left(C_{D S F}\right)^{-1}\left(D S F_{i}-\overline{D S F}\right) .
$$

To quantify the efficiency of the features proposed for damage detection, the number of misclassified points is calculated. The database, composed of time series collected at the undamaged state, and time series collected for various damage levels, is split in three parts :

- the reference baseline, composed of half of the undamaged database. It is used as reference to form the covariance matrix $\left(C_{D S F}\right)$ and mean vector $(\overline{D S F})$ for Mahalanobis distance calculation. 


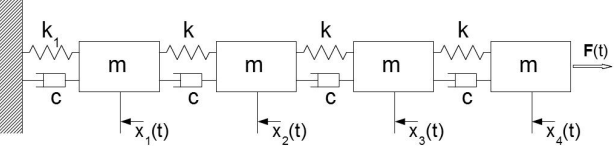

Figure 1. Mass/spring/damper model

- the classification base, composed of a quarter of the undamaged database. This base allows the calculation of a threshold which will be used to classify unknown new vectors. This threshold is based on the standard deviation of Mahalanobis distances of the classification base vectors to the reference base. In this study the threshold is set to 3 standard deviations. If a new vector has a Mahalanobis distance to the reference base higher than the threshold, it is classified as damaged state. Assuming the normal distribution of the feature tested, this implies about $1 \%$ of false alarm accepted.

- the test base, composed of the last quarter of the undamaged database and all the damaged time series. It is used to perform a blind-test aimed at checking the efficiency of each DSF. In this study, since each vector is known, the efficiency of the DSF will be quantified by the number of misclassified vectors, including :

- undamaged state classified as damaged (False Alarm)

- damaged state classified as undamaged (Non-Detection)

\section{Case Studies}

\section{Case Study 1}

The first case study (CS1) is a 4 dof mass/spring/damper numerical model (Fig. 1). The physic constants are selected to be realistic with building structures : $k=2.4 e 8 \mathrm{~N} . \mathrm{m}^{-1}$, $c=1.02 e 6 \mathrm{~N} . \mathrm{m}^{-1} . \mathrm{s}$ and $m=70000 \mathrm{~kg}$. The system can be expressed by the equation

$$
M \ddot{\mathbf{x}}+C \dot{\mathbf{x}}+K \mathbf{x}=\mathbf{f}(t)
$$

where $M, C$ and $K$ represent mass, damping and stiffness $4 \times 4$ matrices, respectively. $\mathbf{f}(t)$ is the excitation vector which applies a white noise at the free end of the model. Equation 15 is solved by a fourth order Runge-Kutta method sampled at $60 \mathrm{~Hz}$. The displacement $x_{1}$, resampled at $30 \mathrm{~Hz}$, is used for damage detection. An example of time series calculated is visible in Fig.2.

Damage is simulated by a decrease in the first spring stiffness, but only when it is in extension (Eq.16) to represent the effect of the closing and opening of a crack.

$$
k_{1}=\left\{\begin{array}{c}
k \text { if } x_{1} \geq 0 \\
(1-\alpha) k \text { if } x_{1}<0
\end{array}\right.
$$

As presented in Tab.1, the database is composed of 120 undamaged time series and 90 damaged time series with an increasing level of damage. 


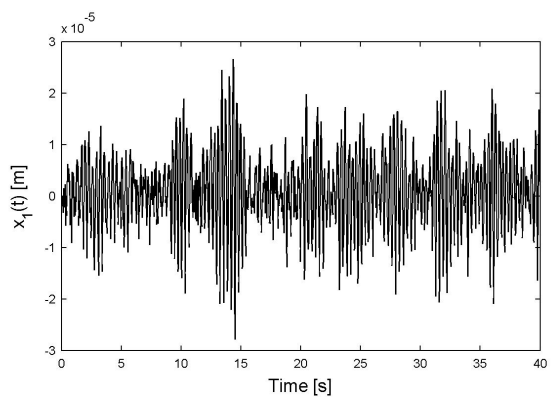

Figure 2. Time series obtained with the model.

Table 1. Composition of the database

\begin{tabular}{ll}
\hline $\begin{array}{l}\text { Number of } \\
\text { time series }\end{array}$ & $\alpha$ \\
\hline 120 & 0 \\
10 & 0.1 \\
10 & 0.2 \\
10 & 0.3 \\
10 & 0.4 \\
10 & 0.5 \\
10 & 0.6 \\
10 & 0.7 \\
10 & 0.8 \\
10 & 0.9 \\
\hline
\end{tabular}

\section{Case Study 2}

For the second case study (CS2), vibration signals of a three story bookshelf structure are considered (Fig.3). These experiments are a part of several case studies carried out by the Los Alamos National Laboratory (LANL) and provided freely for the scientific community. All the informations about the structure and the test set up are available on LANL website [19].

It is composed of Unitrust columns and aluminum floor plates. Support brackets for the columns are bolted to the plates and hold the Unitrust columns. The global geometry is detailed in Fig.4.

A shaker is attached at corner D (Fig.4) in order to excite both torsional and translational vibration modes. Twenty-four accelerometers are positioned on the structure, all in the same direction. The excitation is white noise with several bandwidths from 800 to $3200 \mathrm{~Hz}$, and different input levels from 3,5 to 7 Volts. For each setup, the response is sampled at $1600 \mathrm{~Hz}$ and consists of 8192 points.

For the purpose of testing the sensitivity to damage, only one sensor is considered. It is located at the corner $\mathrm{C}$, on the first floor plate. This is close to the location of the induced damage.

Damage is simulated by changes in the corner $\mathrm{C}$ first floor joint. Tab.2 summarizes 


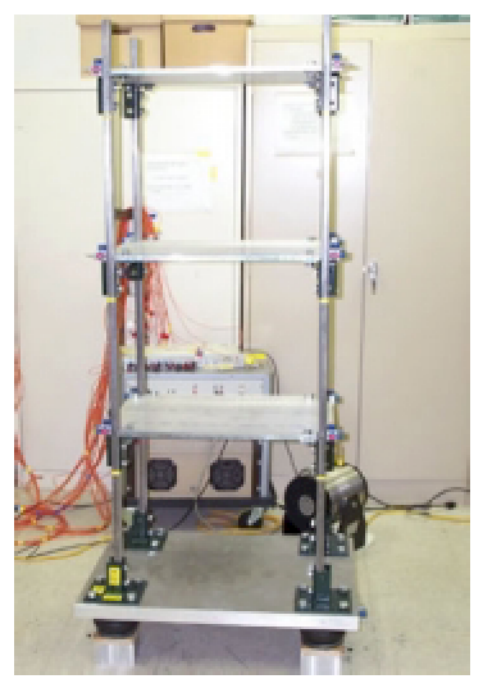

Figure 3. Photo of the test structure (LANL)[19]
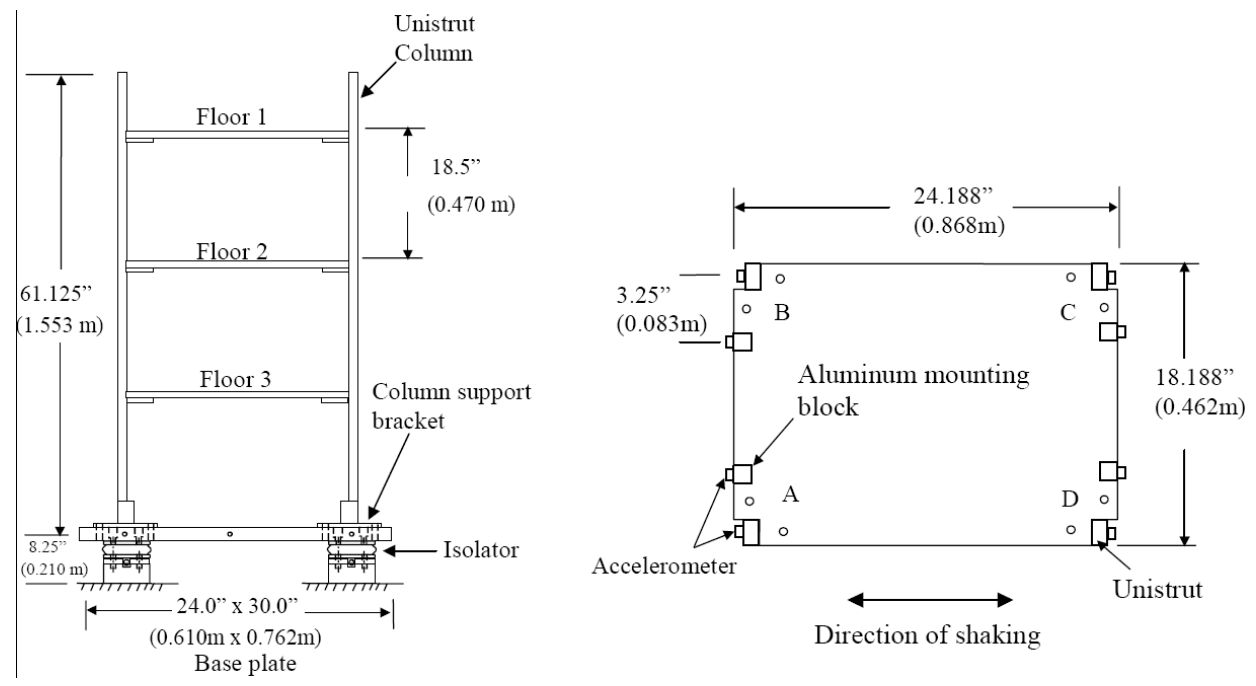

Figure 4. Front (left figure) and top dimensions (right figure) of the test structure $(\mathrm{LANL})[19]$

the different damage cases. The database consists of 150 time series related to the undamaged state, and 60 time series in the damaged state.

\section{Parameters investigated}

In the algorithm described previously, several parameters need to be specified. The first ones are the reconstruction parameters. They are set based on the analysis of baseline series chosen randomly.

The first minimum of the mutual information function and the first zero of the autocorrelation function indicate that a delay of 2 for case study 1, and 6 for case study 2 , are good choices. However, if $T_{2}$ is equal to $\tau$, the two vectors mapped by the polynomial fitting are almost identical leading to identity matrix as Jacobian. Since the 
Table 2. Damage cases : modification of the bolts between the bracket and the plate $(\mathrm{LANL})[19]$

\begin{tabular}{llcc}
\hline Damage Case & Description & $\begin{array}{c}\text { Bolt Torque } \\
{[\mathrm{N.m}]}\end{array}$ & $\begin{array}{c}\text { Number of time } \\
\text { series collected }\end{array}$ \\
\hline 0 & Undamaged & 6.77 & 150 \\
1 & Decrease tightening torque & 1.128 & 15 \\
2 & Decrease tightening torque & 0.564 & 15 \\
3 & hand tighten & - & 10 \\
4 & Bolts removed & - & 10 \\
5 & Bracket removed & - & 10 \\
\hline
\end{tabular}

maximum value investigated is $T_{2}=5$, the choice of the delay is imposed by $T_{2}$ for CS1. Finally, $\tau$ is set to 6 for both case studies.

The false neighbors method indicates that a dimension of 7 for CS1 and CS2 is a good compromise between the stabilization of the number of false neighbors and a small dimension which keeps the calculation time low (Fig.5).

It is important to note that there is no need to have the same embedding parameters for the two case studies.

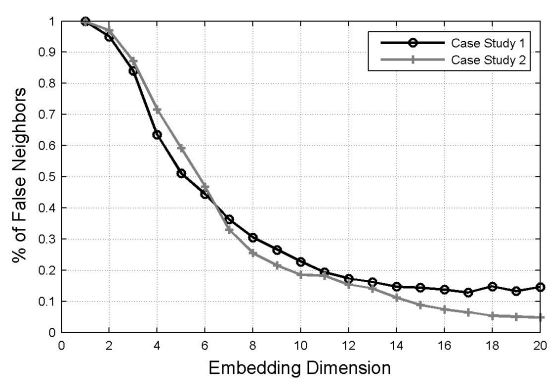

Figure 5. Baseline series \#5: Number of false neighbors functions vs embedding dimension both case studies

For Lyapunov spectrum determination, a preliminary study has shown that the number of transient mapping steps does not exceed 500 and that they are stable after 1500 other ones. These results lead to the choice of $L=2000$ and $L_{0}=500$. Each neighborhood is composed of $N_{\text {para }}$ to $2 N_{\text {para }}$ points, with $N_{\text {para }}$ the number of parameters of the polynomial mapping function to be fitted (depending on the order). At last, 40 fiducial points are followed in each attractor for Lyapunov exponents calculation. For the JFV, since the averaging over the whole attractor is not done according to a large $L$, the number of fiducial points will be 100 .

The order of the polynomial mapping and the mapping steps $\left(T_{2}\right)$ are supposed to be influencing parameters. Therefore, several values are tested. However, the order have to be smaller than 4 to limit the number of points in each neighborhood (329 points for order 4). A similar problem appears with $T_{2}$ in Lyapunov exponents determination, it can not be higher than 3 since the time series are composed of 8192 points and the 
Table 3. Parameters values for DSF calculation

\begin{tabular}{llll}
\hline Feature & $T_{2}$ & $L$ & Order \\
\hline \multirow{2}{*}{ Lyapunov exponents } & 1 & 2000 & $1,2,3$ \\
& 2 & 2000 & 1,2 \\
\hline \multirow{3}{*}{ JFV } & 2 & 2 & $1,2,3$ \\
& $1,2,3$ & 1 & 1 \\
& $1,2,3,4,5$ & 2 & 1 \\
& 2 & $1,2,3,4,5$ & 1 \\
\hline
\end{tabular}

Table 4. Parameters values and percentage of misclassified points in the test-base for Lyapunov exponents

\begin{tabular}{cccc}
\hline$T_{2}$ & Order & \multicolumn{2}{c}{$\%$ of Misclassified points } \\
\cline { 3 - 4 } & & CS1 & CS2 \\
\hline 1 & 1 & 15 & 41 \\
1 & 2 & 23 & 53 \\
1 & 3 & 24 & 62 \\
\hline 2 & 1 & 25 & 61 \\
2 & 2 & 31 & 60 \\
\hline
\end{tabular}

trajectories are followed for 2000 mapping steps. In the case of the JFV, the number of mapping steps before extracting the Jacobian matrix is investigated. Tab. 3 summarizes the values of the parameters involved in the study, leading to 16 parameter setups.

\section{Results and discussion}

\section{Lyapunov Exponents}

The efficiency of Lyapunov Exponents as DSF is very different between the two case studies. For CS2, the percentage of misclassified points varies from $41 \%$ to $62 \%$ (Tab.4) including no false alarms, but a large amont of non-detection (Fig.6b). On the other hand, for CS1, LE are capable of detecting damage from $30 \%-40 \%$ of stiffness reduction, depending on the set of parameters. The poor results obtained in CS2 is caused by the dispersion of the LE values observed on experimental data. This dispersion makes difficult the separation between damaged and undamaged states (Fig.7b).

To determine accurate Lyapunov exponents of chaotic systems, the use of a high order polynomial mapping is preferred. But Tab.4 shows that increasing the order of the mapping leads to more misclassified points. When the polynomial order increases, it is observed that the variations of the different exponents tend to be uniform, leading to a decrease in the sensitivity since each exponent carries the same information (Fig.7). Higher order also produces several positive exponents. Since Lyapunov exponents of noise are theoretically infinite, it probably means that the random part of the time series is modeled by the mapping. The results are not improved by doubling the mapping step. 

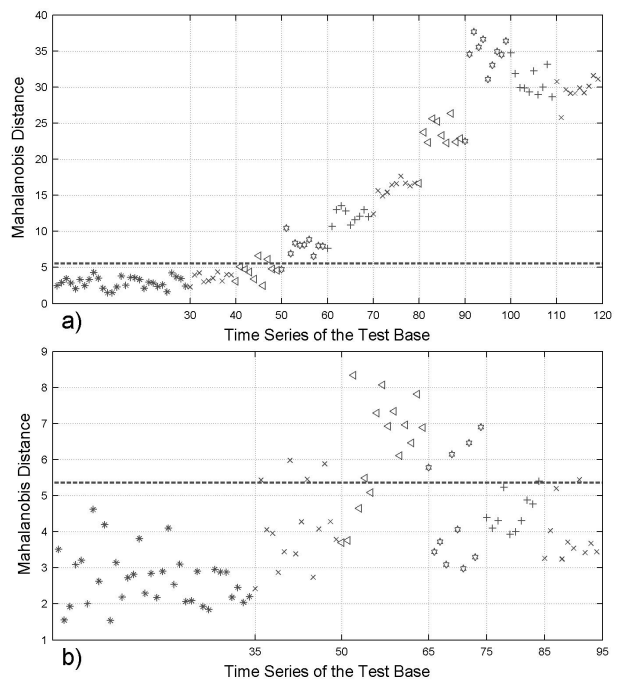

Figure 6. Lyapunov Exponents Mahalanobis distance of the test base for $T_{2}=1$ and linear mapping. The dashed line represents the classification threshold. a) CS1 ; b) CS2 ;

\section{Jacobian Feature Vector}

The number of misclassified time series for the different sets of parameters is presented in Tab.5. Even if some observations will be formulated regarding the influence of the parameter setup, the classification ability is comparable from one setup to another.

For CS1, the worst case counts $51 \%$ of misclassified points and $21 \%$ for the best one, whereas for CS2 the worst case is $16 \%$ which is far better. The change in spring stiffness is not detected before $40 \%$ of reduction, whereas all damage cases are correctly classified for CS2 (Fig.8). This difference is explained by the progressivity of damage introduction in CS1 which make the early detection more challenging.

Looking at the results in details reveals some trends in the parameters influence. Tab.5 shows that increasing the order does not reduce the number of misclassified points. On contrary, it increases for CS2, even if it remains small. The explanation is probably the same as for the Lyapunov exponents. For damage detection, the first order appears more relevant.

The number of misclassified points as $T_{2}$ increases does not present a clear trend. When $L=1$ (setups \#4-5-6), large $T_{2}$ improves the sensitivity for CS1, but not for CS2. Whereas with $L=2$ (setups \#7 to 11), results remain stable, except for $T_{2}=3$ (setup \#9) which will be explained later. This proves that mapping step has no direct effect on the sensitivity to damage, and that it has to be considered in interaction with $L$.

Change in the value of $L$, implies irregular variations in the efficiency of the JFV as presented in Tab.5. For both CS1 and CS2, there are no trends as $L$ increases. However 

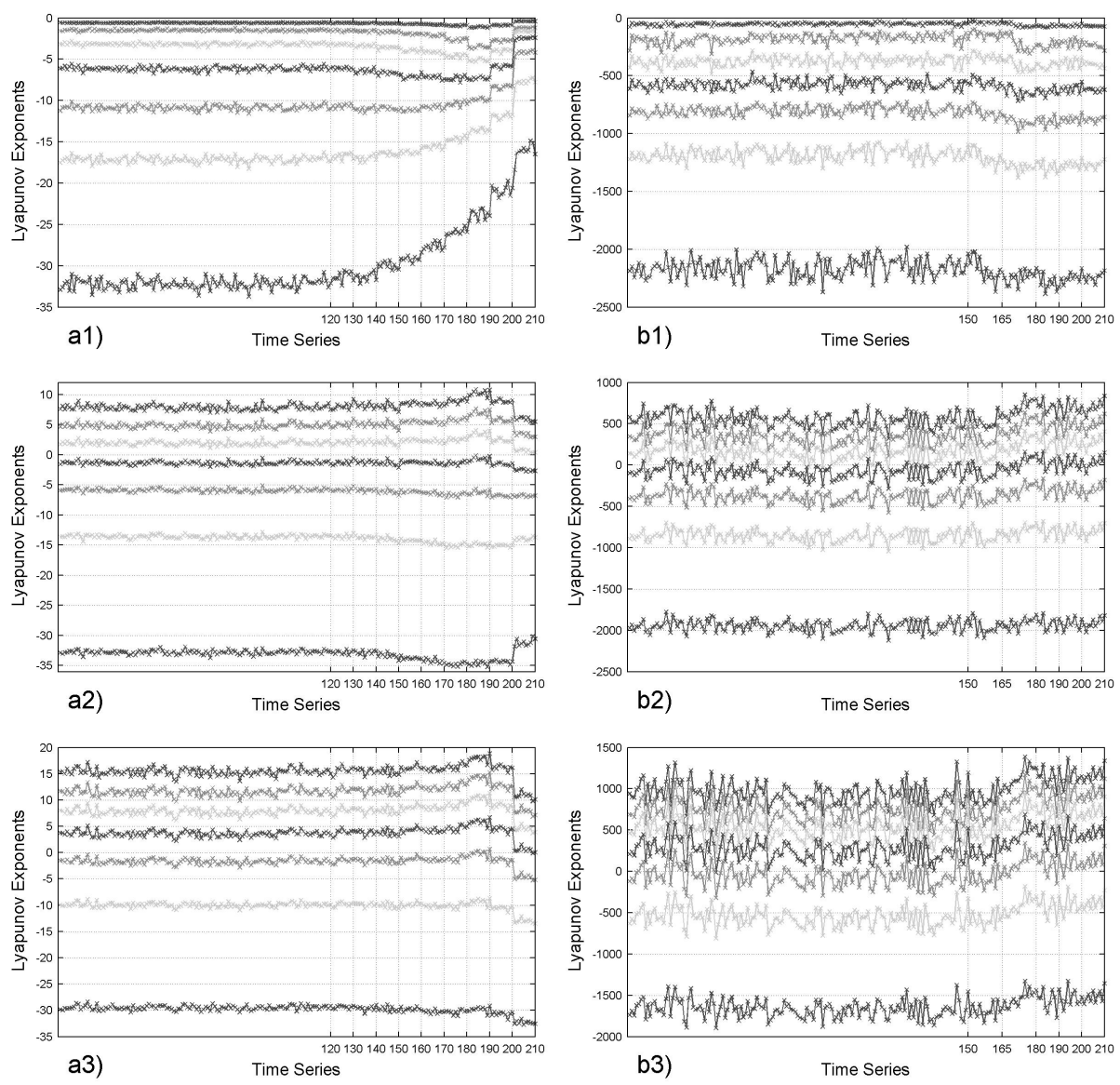

Figure 7. Lyapunov spectrum for the undamaged time series (\#1 to \#150 for CS1 and \#1 to \#120 for CS2) and the damaged time series. Left figure display LE for CS1 and right for CS2. a1) and b1) order=1;a2) and b2) order=2 ; a3) and b3) order=3 ;

the number of misclassified time series is abnormally higher for several $L$ values :

- $L=1$ (setups \#4-5-6 and 12) whatever the value of $T_{2}$

- $L=2$ and $T_{2}=3(\operatorname{setup~\# 9)}$

- $L=3$ and $T_{2}=2(\operatorname{setup~\# 14)}$

- $L=4$ and $T_{2}=2$ (setup \#15).

In order to understand why, for these particular cases, the sensitivity of the JFV is reduced, one has to consider how the different vectors of the state space are reconstructed. The optimal reconstruction delay selected is six time step $(\tau=6)$. The Fig. 9 shows the ten first vectors reconstructed from the time series $(x)=\left\{x_{1}, x_{2}, \ldots, x_{N}\right\}$. In the case $T_{2}=2, L=4$, the Jacobian matrix used in the JFV is calculated between the vectors $X_{7}$ and $X_{9}$ which differ only by one coordinate from initial vector $X_{1}$ and vector $X_{3}$. Therefore this case is equivalent to the case $L=1$ which presents a reduced sensibility. By the same token, the case $T_{2}=2, L=3$ uses a mapping between $X_{5}$ and $X_{7}$. It is close to a mapping between $X_{1}$ and $X_{5}$ formulated with $L=1$ and $T_{2}=4$. 
Table 5. Parameters values and percentage of misclassified points in the test-base for JFV

\begin{tabular}{cccccc}
\hline Set up number & $T_{2}$ & $L$ & Order & \multicolumn{2}{c}{$\%$ Misclassified points } \\
\cline { 4 - 6 } & & & & CS1 & CS2 \\
\hline 1$)$ & & & 1 & 27 & 0 \\
$2)$ & 2 & 2 & 2 & 34 & 1 \\
$3)$ & & & 3 & 34 & 5 \\
\hline 4$)$ & 1 & & & 51 & 14 \\
$5)$ & 2 & 1 & 1 & 43 & 13 \\
$6)$ & 3 & & & 36 & 16 \\
\hline 7$)$ & 1 & & & 30 & 0 \\
$8)$ & 2 & & & 25 & 0 \\
$9)$ & 3 & 2 & 1 & 42 & 9 \\
$10)$ & 4 & & & 28 & 1 \\
$11)$ & 5 & & & 46 & 1 \\
\hline 12$)$ & & 1 & & 46 & 13 \\
$13)$ & & 2 & & 32 & 0 \\
$14)$ & 2 & 3 & 1 & 49 & 4 \\
$15)$ & & 4 & & 30 & 11 \\
$16)$ & & 5 & & 21 & 0 \\
\hline
\end{tabular}
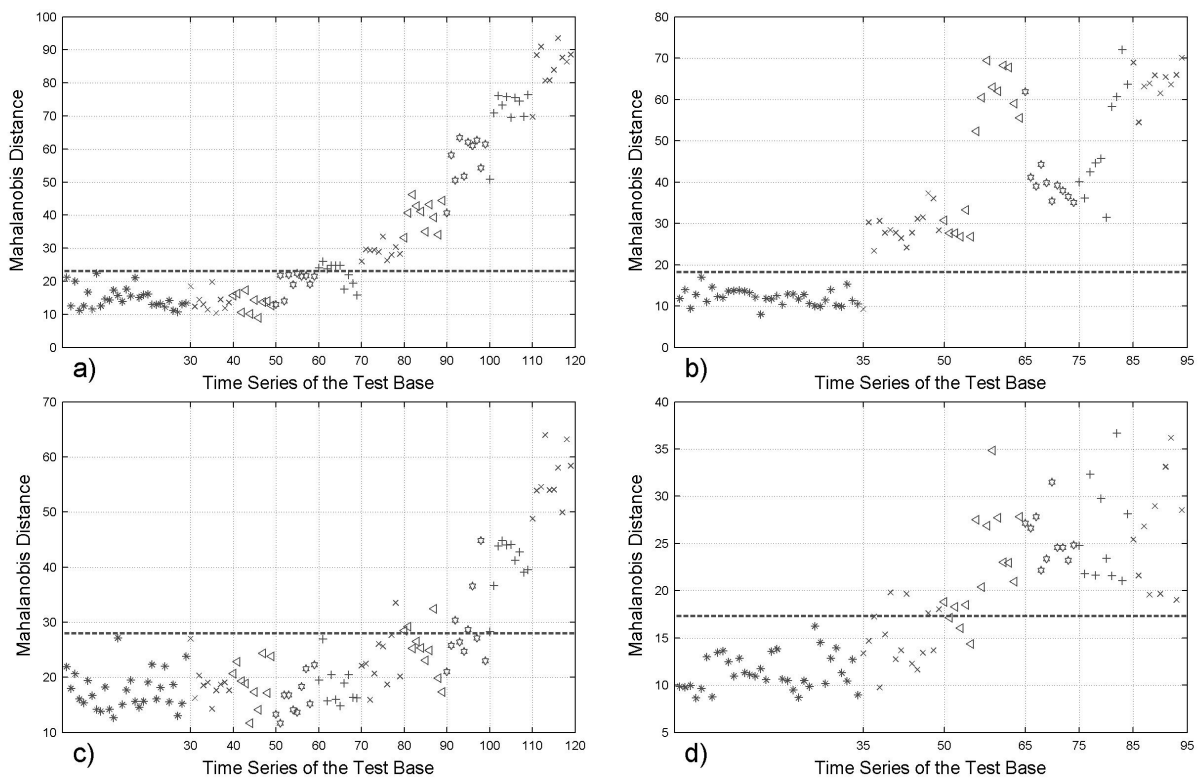

Figure 8. JFV Mahalanobis distance of the test base for the worst and best classification results. The dashed line represents the classification threshold. a) setup \#1 CS1 Best ; b) setup \#1 CS2 Best ; c) setup \#4 CS1 Worst ; d) setup \#4 CS2 Worst.

The same explanation holds for the case $T_{2}=3$ and $L=2$. The Jacobian matrix of the first mapping step $(L=1)$ is less sensitive to damage than the other ones because the initial neighbors are much more close than after few time steps. If the points are spread in a larger area in the attractor, the Jacobian matrix is more able to capture changes 
in the dynamics.

$\begin{array}{cccccccccc}\mathbf{X}_{1} & X_{2} & X_{3} & X_{4} & X_{5} & X_{6} & \mathbf{X}_{7} & X_{8} & X_{9} & X_{10} \\ \boldsymbol{x}_{1} & x_{2} & x_{3} & x_{4} & x_{5} & x_{6} & \boldsymbol{x}_{7} & x_{8} & x_{9} & x_{10} \\ \boldsymbol{x}_{7} & x_{8} & x_{9} & x_{10} & x_{11} & x_{12} & \boldsymbol{x}_{13} & x_{14} & x_{15} & x_{16} \\ \boldsymbol{x}_{13} & x_{14} & x_{15} & x_{16} & x_{17} & x_{18} & \boldsymbol{x}_{19} & x_{20} & x_{21} & x_{22} \\ \boldsymbol{x}_{19} & x_{20} & x_{21} & x_{22} & x_{23} & x_{24} & \boldsymbol{x}_{25} & x_{26} & x_{27} & x_{29} \\ \boldsymbol{x}_{25} & x_{26} & x_{27} & x_{28} & x_{29} & x_{30} & \boldsymbol{x}_{31} & x_{32} & x_{33} & x_{34} \\ \boldsymbol{x}_{31} & x_{32} & x_{33} & x_{34} & x_{35} & x_{36} & \boldsymbol{x}_{37} & x_{38} & x_{39} & x_{40} \\ \boldsymbol{x}_{37} & x_{38} & x_{39} & x_{40} & x_{41} & x_{42} & \boldsymbol{x}_{43} & x_{44} & x_{45} & x_{46}\end{array}$

Figure 9. Ten first reconstructed vectors.

\section{Discussion}

The sensitivity of the JFV depends on the set of parameters chosen. As explained previously, for an optimal sensibility, it is important to avoid the inclusion of the initial neighborhood (and its reconstruction multiples) in the Jacobian calculation for the JFV. This condition is verified if

$$
\begin{aligned}
& T_{2} \cdot L \neq \tau \text { and } \\
& T_{2} \cdot L \neq \tau-L \text { with } L>1
\end{aligned}
$$

Keeping $T_{2}$ as small as possible will contribute to reduce the processing time. In this study, this is achieved with (Fig.8a et Fig.8c) :

- linear mapping

- trajectories followed for two mapping steps $(L=2)$

- mapping steps formed by one or two time steps $\left(T_{2}=1,2\right)$.

Once these conditions are verified, the JFV is able to detect damage quite effectively. For the CS1, in which data are obtained with a numerical model, the efficiency of the LE is slightly better than the JFV since $30 \%$ of stiffness reduction is detected by LE, against $40 \%$ by the JFV. However, on real data, LE hardly detect the damage introduced on the bookshelf structure, whereas all cases are correctly identified by JFV. It is likely that time series in CS2 contain some noise due to experimental conditions which is not present in the simulated data. It seems that JFV is more robust to noise than LE.

Moreover, to calculate the Lyapunov spectrum of a time series with an Intel ${ }^{\circledR}$ Xeon $3 \mathrm{GHz}$ processor, the CPU time varies from 170s to 3700 s depending the polynomial order, whereas it is only between $0.2 \mathrm{~s}$ to $6 \mathrm{~s}$ for the new JFV.

The study shows that the proposed feature is an interesting alternative to Lyapunov exponents for SHM purpose, regarding both sensitivity to damage and computation time.

\section{Conclusion}

Using the full Lyapunov spectrum as damage sensitive feature is very time consuming. To overcome this issue, the Jacobian Feature Vector (JFV), a new damage sensitive 
feature based on the Jacobian matrix of reconstructed dynamics in the state space, is proposed. Instead of following the trajectories for several thousand time steps, as needed for Lyapunov Exponents (LE) calculation, the JFV involves the Jacobian matrix at the early time steps. Both JFV and LE are compared to detect damage on a simulated mass/spring model and a laboratory bookshelf structure. Unlike LE, the JVF is able to detect the damage introduced in the two case studies with an appropriate set of parameters. This indicates that the proposed approach is more robust than LE. Future investigations will be focused on :

- the verification of the sensibility to damage with variable signal-to-noise ratios

- other case studies

- the comparison of the JFV approach to common SHM paradigms (modal analysis, autoregressive models etc.).

\section{Acknowledgments}

The authors gratefully acknowledge the Los Alamos National Laboratory for the data provided to the scientific community and the Ministère de l'Enseignement Supérieur et de la Recherche for its funding.

\section{References}

[1] J.M. Nichols, M.D. Todd, and J.R. Wait. Using state space predictive modeling with chaotic interrogation in detecting joint preload loss in a frame structure experiment. Smart Materials and Structures, 12(4):580-601, 2003.

[2] M.D. Todd, J.M. Nichols, L.M. Pecora, and L.N. Virgin. Vibration-based damage assessment utilizing state space geometry changes: local attractor variance ratio. In Smart Materials and Structures, pages 1000-1008.

[3] J. M. Nichols. Structural health monitoring of offshore structures using ambient excitation. Applied Ocean Research, 25(3):101-114, June 2003.

[4] C.C. Olson, L.A. Overbey, and MD Todd. Sensitivity and computational comparison of statespace methods for structural health monitoring. In Proceedings of SPIE, volume 5768, page 241, 2005.

[5] S. Ghafari, F. Golnaraghi, and F. Ismail. Effect of localized faults on chaotic vibration of rolling element bearings. Nonlinear Dynamics, 53(4):287-301, 2008.

[6] R.A. Livingston, S. Jin, and D. Marzougui. Application of nonlinear dynamics analysis to damage detection and health monitoring of highway structures. In Proceedings of SPIE - The International Society for Optical Engineering, volume 4337, pages 402-410, Newport Beach,CA, 2001. Society of Photo-Optical Instrumentation Engineers.

[7] Fabio Casciati and Sara Casciati. Structural health monitoring by lyapunov exponents of nonlinear time series. Structural Control and Health Monitoring, 13(1):132-146, 2006.

[8] H. Kantz and T. Schreiber. Nonlinear Time Series Analysis. Cambridge University Press, 2004.

[9] V.I. Oseledec. A multiplicative ergodic theorem: Lyapunov characteristic numbers for dynamical systems. Trans. Moscow Math. Soc, 19:197-231, 1968.

[10] Henry D. I. Abarbanel, Reggie Brown, John J. Sidorowich, and Lev Sh. Tsimring. The analysis of observed chaotic data in physical systems. Rev. Mod. Phys., 65(4):1331-, October 1993.

[11] H.D.I. Abarbanel, R. Brown, and MB Kennel. Local lyapunov exponents computed from observed data. Journal of Nonlinear Science, 2(3):343-365, 1992. 
[12] F. Takens. Detecting strange attractors in turbulence. Dynamical systems and turbulence, Warwick 1980, pages 366-381, 1980.

[13] Andrew M. Fraser and Harry L. Swinney. Independent coordinates for strange attractors from mutual information. Physical Review A, 33(2):1134-1140, February 1986.

[14] Reggie Brown, Paul Bryant, and Henry D. I. Abarbanel. Computing the lyapunov spectrum of a dynamical system from an observed time series. Phys. Rev. A, 43(6):2787-, March 1991.

[15] M. Sano and Y. Sawada. Measurement of the lyapunov spectrum from a chaotic time series. Physical Review Letters, 55(10):1082-1085, 1985.

[16] J.P. Eckmann, S.O. Kamphorst, D. Ruelle, and S. Ciliberto. Liapunov exponents from time series. Physical Review A, 34(6):4971-4979, 1986.

[17] K. Worden, C.R. Farrar, G. Manson, and G. Park. The fundamental axioms of structural health monitoring. Proceedings of the Royal Society A: Mathematical, Physical and Engineering Science, 463(2082):1639, 2007.

[18] K. Worden, G. Manson, and N. R. J. Fieller. Damage detection using outlier analysis. Journal of Sound and Vibration, 229(3):647-667, January 2000.

[19] Los Alamos National Laboratory. The bookshelf structure. http://institute.lanl.gov/ei/. 